\title{
Diseño y desarrollo de una plataforma microfluídica con electrodos interdigitados para espectroscopía por impedancia eléctrica
}

\section{Design and development of a microfluidic platform with interdigitated electrodes for electrical impedance spectroscopy}

\author{
José Miguel Barboza-Retana', Cristopher Vega-Sánchez², \\ Juan J. Rojas ${ }^{3}$, Steven Quiel-Hidalgo ${ }^{4}$, Sofia Madrigal-Gamboa ${ }^{5}$, \\ Paola Vega-Castillo ${ }^{6}$, Renato Rímolo-Donadío ${ }^{7}$
}

Fecha de recepción: 22 de setiembre de 2020

Fecha de aprobación: 8 de enero de 2021

Barboza-Retana, J.M; Vega-Sánchez, C; Rojas, J.J; QuielHidalgo, S; Madrigal-Gamboa, S; Vega-Castillo, P; RímoloDonadío, R. Diseño y desarrollo de una plataforma microfluídica con electrodos interdigitados para espectroscopía por impedancia eléctrica. Tecnología en Marcha. Vol. 35-1. Enero-Marzo 2022. Pág 54-66.

doi) https://doi.org/10.18845/tm.v35i1.5389

1 Profesor de la Escuela de Ingeniería Electrónica, Instituto Tecnológico de Costa Rica. Costa Rica. Correo electrónico: jmbarboza@itcr.ac.cr (iD) https://orcid.org/0000-0003-0333-7904

2 Profesor de la Escuela de Ingeniería Electromecánica. Instituto Tecnológico de Costa Rica. Costa Rica. Correo electrónico: cvega@itcr.ac.cr (D) https://orcid.org/0000-0002-2174-8291

3 Profesor de la Escuela de Ingeniería Electromecánica. Instituto Tecnológico de Costa Rica. Costa Rica. Correo electrónico: juan.rojas@itcr.ac.cr (iD) https://orcid.org/0000-0002-3261-5005

4 Estudiante de Ingeniería en Mecatrónica. Instituto Tecnológico de Costa Rica. Costa Rica. Correo electrónico: stevenquiel@estudiantec.cr (iD https://orcid.org/0000-0001-6767-8463

5 Estudiante de Ingeniería en Mecatrónica. Instituto Tecnológico de Costa Rica. Costa Rica. Correo electrónico: smadrigal.sc@gmail.com (iD https://orcid.org/0000-0002-2990-5909

6 Profesora de la Escuela de Ingeniería Electrónica. Instituto Tecnológico de Costa Rica. Costa Rica. Correo electrónico: pvega@itcr.ac.cr

7 Profesor de la Escuela de Ingeniería en Electrónica. Instituto Tecnológico de Costa Rica. Costa Rica. Correo electrónico: rrimolo@itcr.ac.cr 


\section{Palabras clave}

Análisis espectral; Analizador Vectorial de Redes (AVR); Espectroscopia por Impedancia Eléctrica (EIS); microfluídica; microsistemas; sensores.

\section{Resumen}

En este artículo se describe el proceso de diseño, fabricación y caracterización de un arreglo de sensores para realizar EIS de amplio espectro para muestras en solución acuosa en una plataforma microfluídica. Su capacidad de amplio aspecto permitirá desarrollar estudios de caracterización de fluidos con resultados que no son posibles de obtener en sistemas de menor rango de frecuencia. El sistema microfluídico consta de cuatro cámaras de llenado y los canales de transporte de fluidos requeridos para la purga y llenado. En la sección inferior de cada cámara hay un electrodo depositado en oro que permite la interacción eléctrica con la muestra. La geometría de las cámaras y los canales transporte y de los electrodos fueron diseñadas utilizando un proceso de optimización por medio de simulaciones multifísicas. Luego, se desarrollaron experimentalmente diferentes procesos de fabricación para SU8 y PDMS los cuales permitieron llevar a cabo la implementación del sistema microfluídico. Finalmente, utilizando una interfaz de comunicación eléctrica diseñada para la interconexión del sistema microfluídico con un analizador vectorial de redes, se desarrollaron las mediciones requeridas para obtener el comportamiento de cada sensor.

Los resultados obtenidos mostraron un comportamiento predominantemente capacitivo de cada sensor desde bajas frecuencias hasta $2 \mathrm{GHz}$, permitiendo obtener suficiente contraste entre las diferentes soluciones acuosas medidas, logrando una buena repetitividad entre las mediciones realizadas en las distintas cámaras.

\section{Keywords}

Electrical Impedance Spectroscopy (EIS); microfluidics; microsystems; sensors; spectral analysis; Vector Network Analyzer (VNA).

\footnotetext{
Abstract

In this paper, we describe the design, manufacturing, and characterization of a sensor array suitable for implementing wide broad band EIS for samples in aqueous solution on a microfluidic platform. Its wide-ranging capacity will allow the development of fluid characterization studies with results that are not possible to obtain in systems with a lower frequency range. The microfluidic system consists of four filling chambers and the fluid transport channels required for flushing and filling. At the bottom section of each chamber there is a gold-deposited electrode that allows electrical interaction with the sample. The chamber's geometry, transport channels, and the electrodes were designed using an optimization process through multiphysics simulations. Then, different manufacturing processes were developed experimentally for SU8 and PDMS which allowed to carry out the implementation of the microfluidic system. An electrical communication interface was designed for the interconnection of the microfluidic system with the vector network analyzer (VNA). Then, the measurements required to obtain the behavior of each sensor were developed.

The results obtained show a predominant capacitive behavior of each sensor from low frequencies up to $2 \mathrm{GHz}$, allowing to obtain sufficient contrast between different aqueous solutions and a good repeatability in different sensing chambers with the same solutions.
} 


\section{Introducción}

La espectroscopía de impedancia eléctrica (EIS, de sus siglas en inglés, Electrical Impedance Spectroscopy) es una técnica a través de la cual se pueden obtener las firmas espectrales características en términos de impedancia de muy diversos materiales y compuestos. Este método es aplicable a múltiples escenarios en áreas de la biotecnología y la medicina: pruebas toxicológicas [1]-[2], diagnóstico médico [3]-[6], investigación básica de propiedades celulares, viabilidad y concentración celular, caracterización de tejidos [7] caracterización de biomasa en procesos biotecnológicos [8]-[9], estudio de células madre, pruebas de medicamentos [10] y terapias químicas personalizadas [11], por mencionar algunas.

Como alternativa a los métodos de marcadores de fluorescencia u otras técnicas semejantes, que son destructivas sobre las muestras, la EIS constituye un método de bajo costo, no invasivo y que puede proporcionar datos en tiempo real sin dañar la muestras, constituyendo una herramienta valiosa y versátil [12]. Otra aplicación consiste en la estimulación eléctrica de cultivos celulares, la cual puede ser implementada nativamente a través de la aplicación de campos eléctricos de magnitud variable a las muestras [13].

Existen diversas formas de implementar EIS desde la perspectiva del procesamiento electrónico, tales como mezcla y filtrado (lock-in), oscilación, o muestreo síncrono [14]-[15], y para todas esas arquitecturas es necesario contar con un elemento transductor que permite aplicar una señal eléctrica a la muestra bajo prueba para así medir su impedancia. Este elemento se implementa generalmente como una cámara donde se disponen electrodos metálicos que permiten inyectar y medir la señal eléctrica, la cual debe ser función de las propiedades del material dentro de dicha cámara [16].

Muchas de las soluciones de ingeniería desarrolladas para implementar la técnica de EIS permiten abarcar pruebas en un rango de baja y media frecuencia, siendo a lo sumo algunos cuantos $\mathrm{MHz}$ [17]-[19]. Sin embargo, muchas de las aplicaciones requeridas para el estudio celular requieren de soluciones que permitan la aplicación de EIS en rangos de mucha mayor frecuencia, siendo necesario frecuencias de medición en el orden de los GHz [20]-[21]. Aún así, los retos de ingeniería necesarios de resolver para disponer de un sistema EIS de amplio espectro ha implicado que no existan muchas soluciones con dichas características. Algunos sistemas desarrollados han permitido llegar a frecuencias máximas de $3 \mathrm{GHz}$ [20], $9 \mathrm{GHz}$ [21] y $40 \mathrm{GHz}[22]$.

En este trabajo se aborda el proceso de diseño de un arreglo de sensores y la plataforma microfluídica que permiten hacer el proceso de transducción a pequeña escala para muestras en suspensión acuosa, desde su diseño conceptual, simulaciones multifísicas para su dimensionamiento y optimización, así como la fabricación y caracterización reológica y eléctrica. En la figura 1 se muestra el diseño conceptual con la integración de los componentes requeridos para implementar un sistema de EIS completo. 


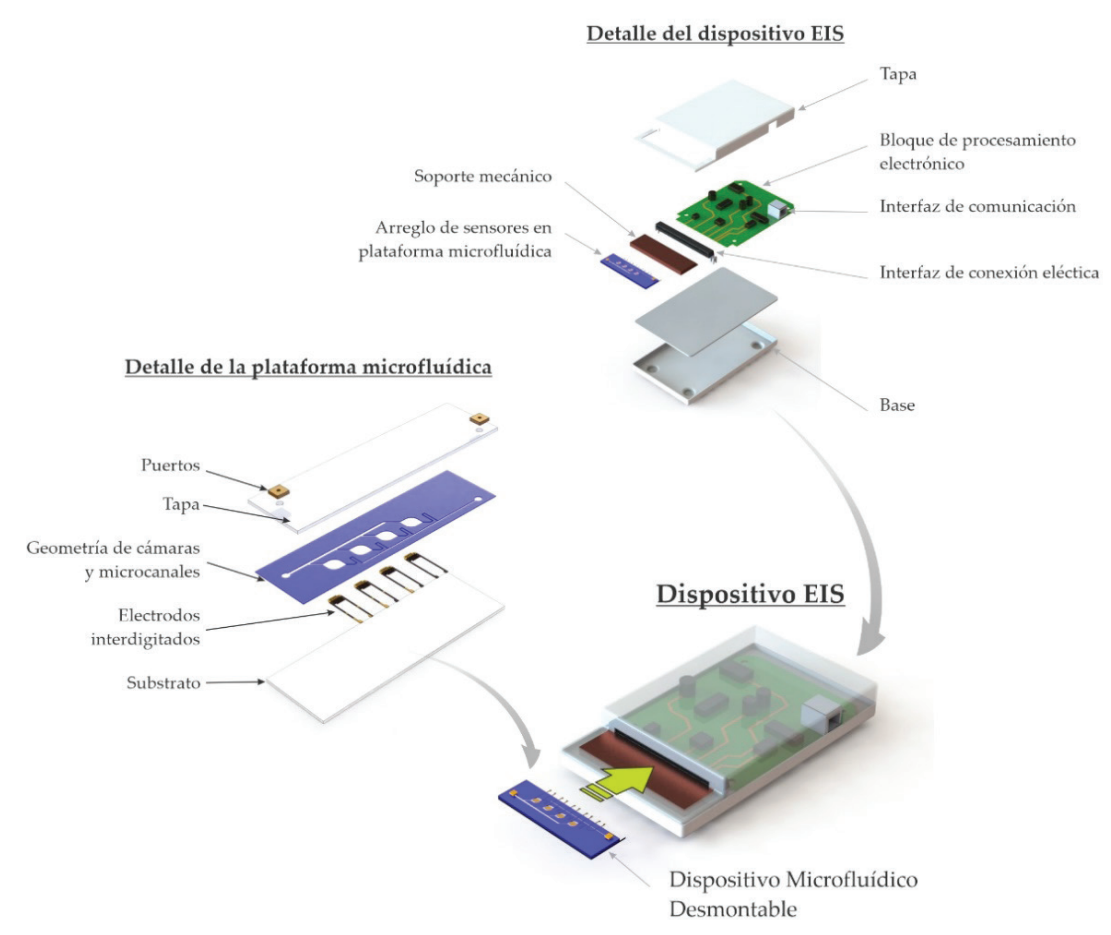

Figura 1. Diagrama conceptual de los componentes de un sistema de EIS que contempla el arreglo de sensores descrito en este artículo.

\section{Diseño del arreglo de sensores}

La estructura básica del sensor para EIS presentada en este trabajo se forma con un soporte sobre el cual se depositan electrodos de oro, los cuales se cubren con una capa con microfluídica que contiene la cámara de sensado, donde se fluye el material a analizar por medio de los canales para la infusión y perfusión de la cámara. El diseño de la estructura del sensor se basó en trabajos previos en la literatura, particularmente los propuestos por Sugiura et al. [23] y Chen et al. [22]. El diseño geométrico utilizado por Sugiura et al. en [23] se consideró como base para el desarrollo del sistema microflú́dico debido a que uno de los requerimientos propuestos en esta solución era que el sistema permitiera ejecutar al menos mediciones sobre 4 muestras simultáneas, permitiendo así que cada una de ellas experimentara las mismas condiciones de temperatura, presión y tiempo de exposición. Además, para la selección de materiales y técnicas de fabricación para el sistema microfluídico se tomó como apoyo lo reportado por X. $\mathrm{Ma}$ et al. en [20]. Otros estudios recientes han desarrollado soluciones similares desde el punto de vista de la fabricación, uno de ellos el reportado por R. Bounik et al. en [19], donde utilizan el PDMS puede reproducir estructuras en el orden de los cientos de nanómetros presentes en un molde, representando un material con un gran potencial, además de ser un material transparente permitiendo la visualización del fluido al trasportarse por el dispositivo.

Para cumplir el requisito de que el dispositivo fuese de bajo costo, se decidió utilizar como substrato un portaobjeto estándar de vidrio con dimensiones de $26 \mathrm{~mm} \times 76 \mathrm{~mm} \times 1 \mathrm{~mm}$, con la geometría final que se indica en la figura 2. El volumen total de fluido que puede alojar el

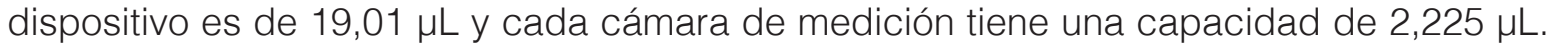




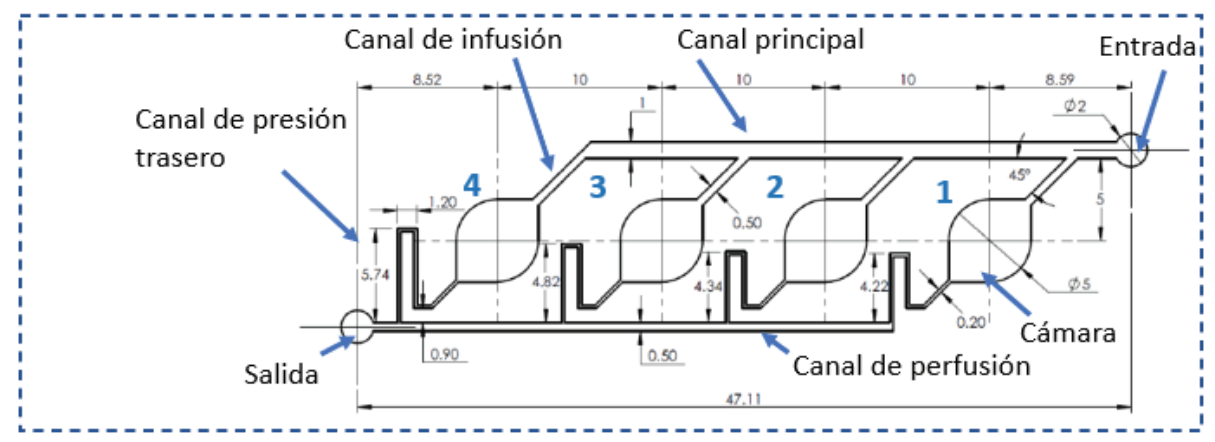

Figura 2. Geometría y dimensiones del arreglo de sensores propuesto, con cuatro cámaras (no se muestran los electrodos, ni la estructura de extracción). Escala en mm.

El proceso se acompañó en una primera fase por exhaustivos estudios a nivel de simulación con las herramientas Comsol Multiphysics, Ansys Fluent y Ansys Electromagnetic Desktop (HFSS). Con estas herramientas se hicieron estudios del comportamiento hidráulico de las cámaras y de los canales de transporte. En esos estudios se trabajó con la optimización de las dimensiones geométricas del sistema microfluídico con la meta de permitir el transporte homogéneo de fluido a lo largo de todo el canal de infusión y con ello el llenado completo de cada cámara, considerando magnitudes de presión, velocidad, esfuerzo cortante y líneas de flujo controladas. La figura 3 muestra la estructura base para cada cámara de sensado y el electrodo interdigitado final, así como la respuesta simulada de impedancia obtenida con la biblioteca de HFSS de ANSYS. El resultado obtenido muestra un comportamiento capacitivo, permitiendo distinguir materiales con diferentes constantes dieléctricas ubicados dentro de la cámara. Por las dimensiones del sensor, las líneas de acceso en el sensor y la tarjeta de interfaz ejercen un efecto para frecuencias en el rango $\mathrm{GHz}$, las cuales se remueven por medio de una estrategia de extracción, asunto que se detalla posteriormente.

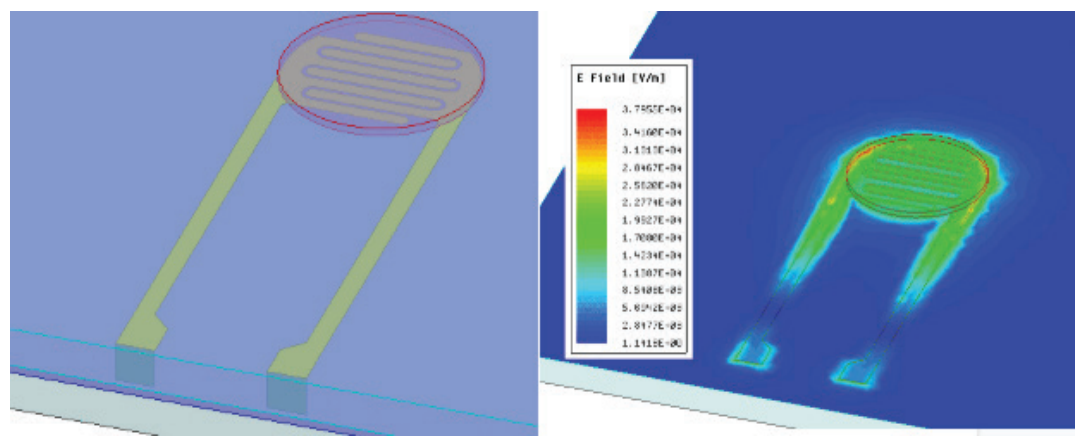

(a)

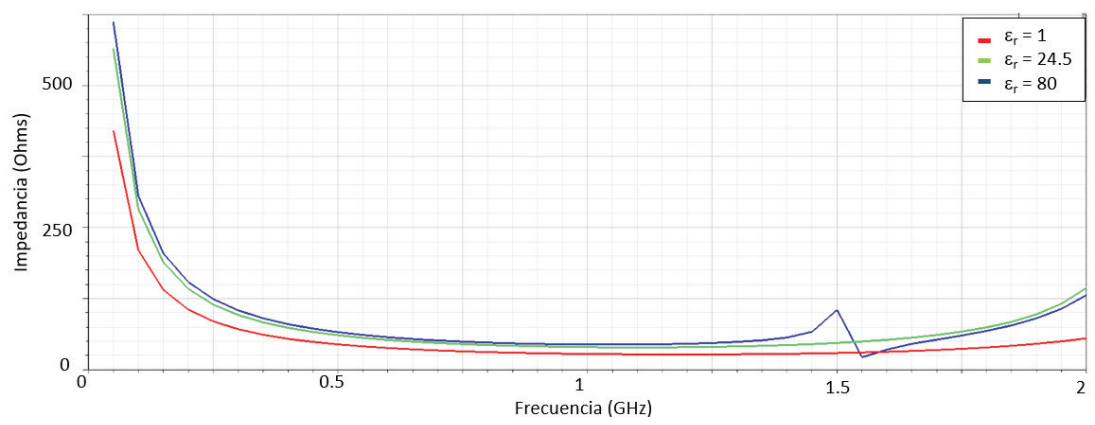

(b)

Figura 3. Modelo de simulación electromagnético para el sensor de EIS: (a) geometría base que muestra los electrodos y la distribución de campo eléctrico a $2 \mathrm{GHz}$; (b) respuesta en frecuencia de impedancia simulada para el sensor considerando diferentes medios en la cámara. 

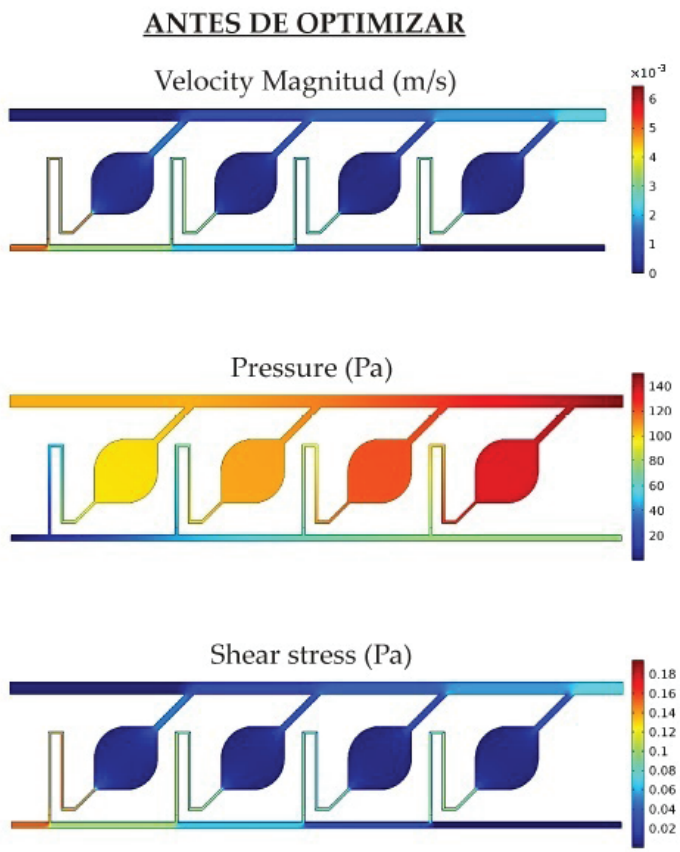

Streamline velocity field

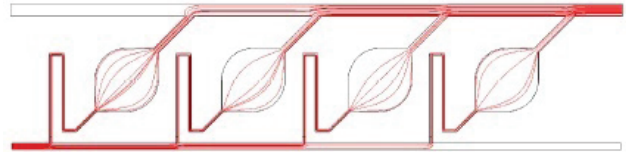

(a)

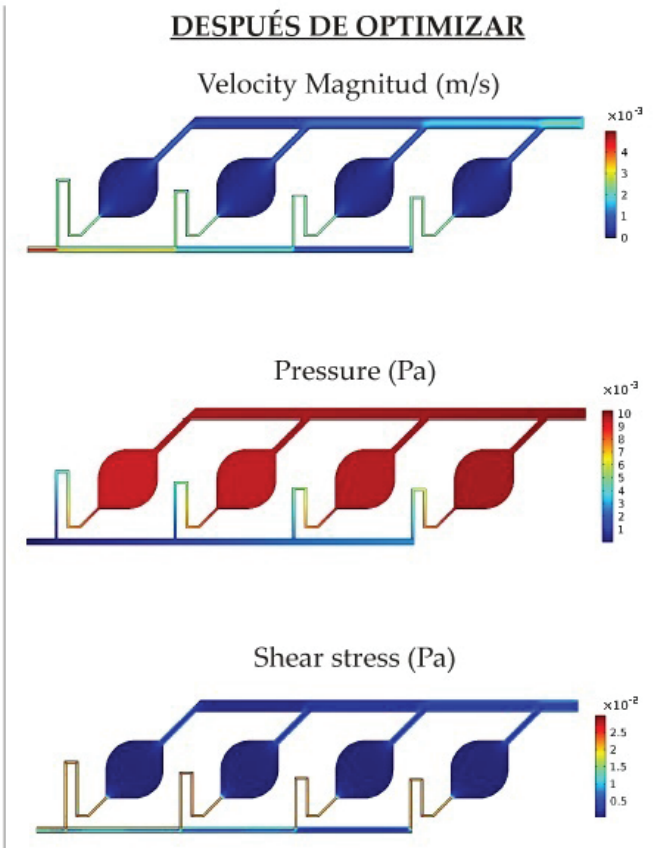

Streamline velocity field

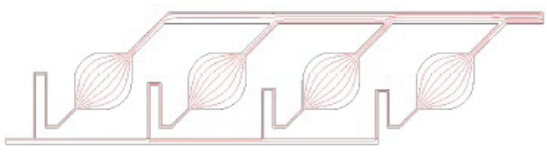

(b)

Figura 4. Proceso de optimización del diseño a nivel de simulación: velocidad, presión, tensión y campo de velocidad del arreglo de sensores antes (a) y luego (b) de la optimización geométrica.

En la figura 4 se ilustra el proceso de optimización del balance del caudal en cada cámara, donde se muestran las distribuciones de velocidad, esfuerzo cortante y presión antes de optimizar (desiguales) y luego de su optimización (balanceadas). La optimización se desarrolló modificando la longitud del canal de salida de flujo de cada cámara, permitiendo que la resistencia dinámica resultante ajustara el balance requerido. Este detalle se puede observar en la figura 4, donde el resultado final (derecha) utiliza distintas longitudes en cada una de las cámaras. Así, la importancia del balance logrado radica en: 1) garantizar un llenado completo (sin burbujas) del dispositivo que podría alterar las mediciones de impedancia eléctrica y, 2) garantizar un mismo caudal a través de las cuatro cámaras en caso de que se desee realizar mediciones dinámicas en condiciones de flujo constante.

\section{Proceso de fabricación}

El sistema final integrado por el arreglo de sensores junto con los canales y cámaras de llenado, así como los electrodos y la estructura de extracción, se observa en la figura 5, para el cual se sigue un proceso de fabricación en el que se involucran una serie de técnicas utilizadas en la construcción de sistemas microfluídicos. 


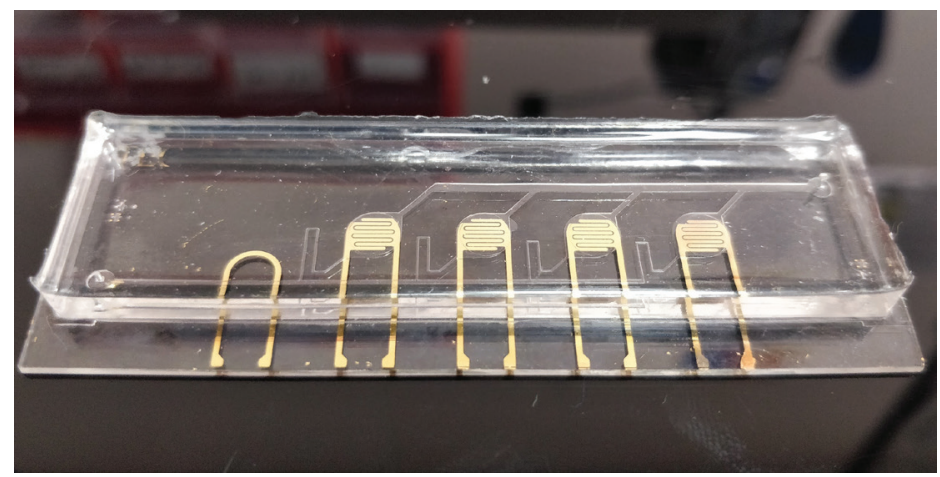

Figura 5. Arreglo de sensores integrado con las cámaras de llenado y los canales de infusión y perfusión.

En la etapa inicial de la fabricación, se toma un substrato de vidrio y se somete a un proceso de limpieza profunda por medio distintas soluciones como piraña (H2SO4 activado con H2O2), sulfocrómica, acetona e isopropanol. En este proceso de limpieza se extrema el cuidado con el fin de eliminar toda impureza de la superficie de los vidrios y de no contaminar los mismos con partículas del ambiente. Así, finalizado este proceso se hace una deposición de una capa de fotoresistivo S1805. Esta película se expone a luz ultravioleta (UV) utilizando foto máscaras con la geometría de los electrodos con el fin de lograr la activación del material en las zonas requeridas. Luego, se realiza el revelado con la solución Microposit-MF321, la cual permite eliminar el fotoresistivo S1805 no expuesto a la UV. Este proceso permite disponer la preparación para la deposición de los electrodos. Para ello, se deposita una primera película de titanio de $67 \AA$ para mejorar la adherencia y finalmente una capa de oro de un grosor de $541 \AA$, utilizando la técnica de PVD (physical vapor deposition). Una vez concluida la deposición, los substratos se llevan a un proceso de decapado (stripping) con acetona que permite remover todas las secciones de oro no requeridas para lograr revelar la geometría de cada electrodo.

En la siguiente etapa se siguió el mismo tratamiento de limpieza para substratos de vidrio mencionado, esto con el fin de proceder a la creación de los moldes con el fotoresistivo SU82100 utilizados para la fabricación de los canales y cámaras del sistema de llenado con PDMS (polidimetilsiloxano). Con el molde de SU8-2100 se dispone una cámara especial de vertido donde se llena de PDMS con el fin de que este tome la forma requerida de los canales y cámaras, según se observa en la figura 5.

Finalmente, utilizando una capa muy fina de PDMS sin curar se realiza la unión (bonding) entre el substrato con los sensores y las cámaras-canales del PDMS. La fina capa de PDMS hace el trabajo de una goma para lograr la adherencia entre partes. Para aumentar la fuerza de unión se utiliza un sistema de prensado mecánico que permite aplicar una presión constante sobre toda el área de unión mientras la capa de PDMS toma las condiciones de secado y consistencia final.

\section{Caracterización del arreglo de sensores}

La interfaz de comunicación para conectar el arreglo de sensores y la microfluídica con el analizador de red vectorial (VNA de sus siglas en inglés, vector network analyzer) se realizó a través de una tarjeta de circuito impreso. Esta tarjeta se diseñó según características que permitieran la integridad en las mediciones en alta frecuencia. Entre estas características se tomó en consideración un diseño de pistas eléctricas con una impedancia característica de $50 \Omega$ y con una distancia física de $44 \mathrm{~mm}$ para cada sensor desde el ingreso de la señal por el conector SMA (SubMiniature version A) hasta el punto de conexión con el electrodo. En la figura 6 se muestra la tarjeta de interconexión luego de ser fabricada y ensamblada. Para este 
sistema se utilizaron conectores SMA de alto desempeño en frecuencia para un rango máximo de $20 \mathrm{GHz}$. Para realizar la conexión eléctrica entre las pistas del PCB y los sensores se utilizó una resina epóxica conductora en base a partículas de plata $(\mathrm{Pt})$.

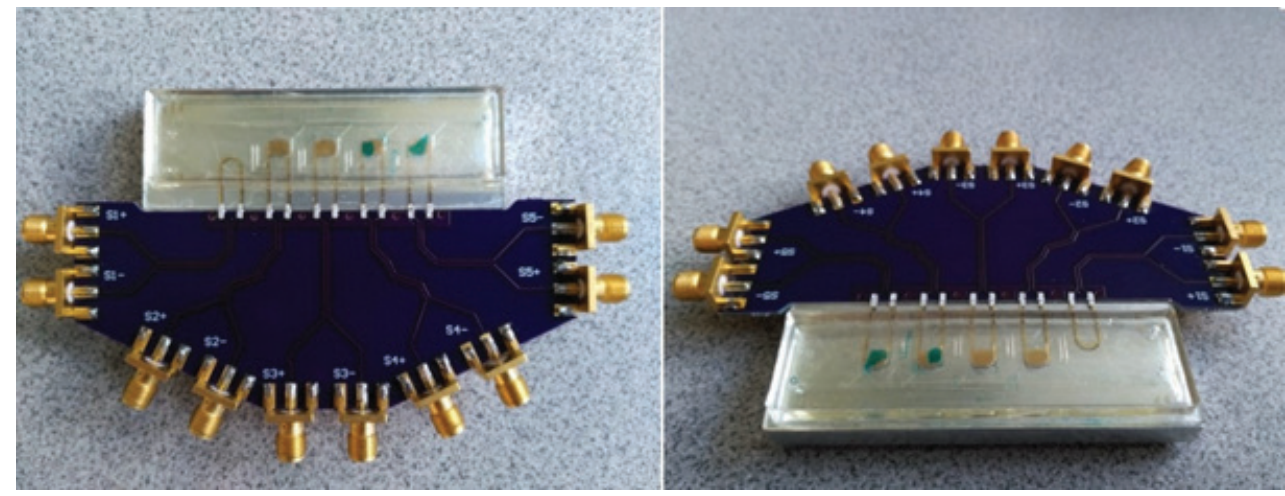

Figura 6. Sistema integrado para medición de espectroscopía por medio del VNA.

Con el sistema anterior se realizaron las pruebas experimentales utilizando el VNA. En la Figura 7 se observa el sistema final de pruebas, según el cual se planteó el procedimiento para la obtención de espectroscopía por impedancia eléctrica para medios acuosos.

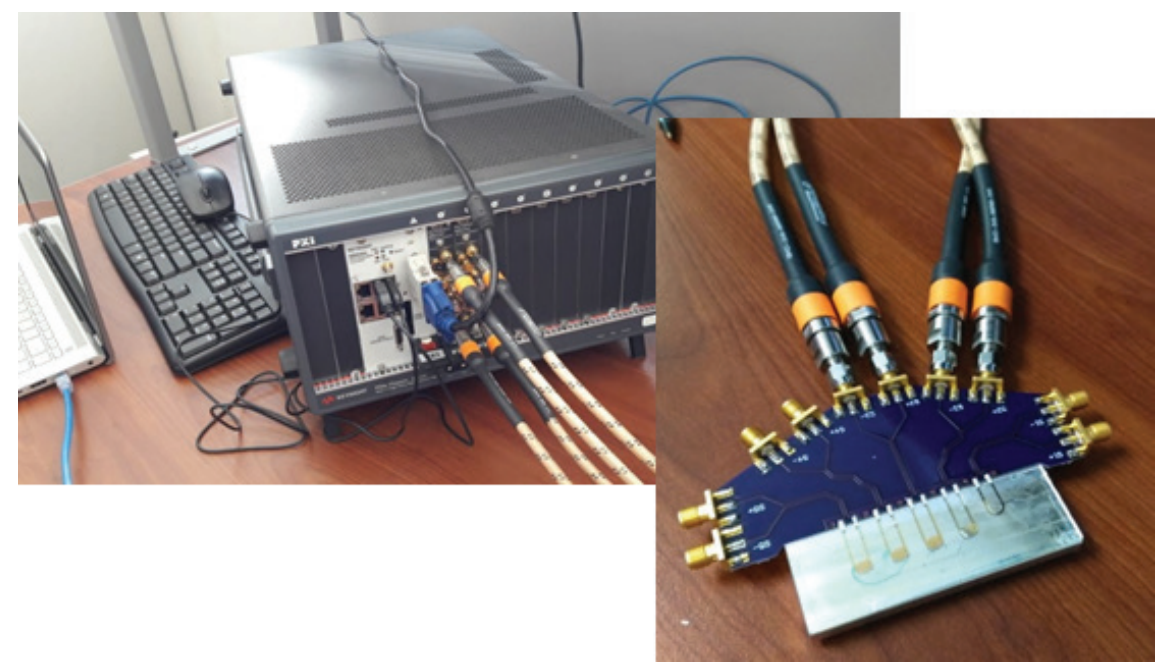

Figura 7. Sistema integrado para medición de espectroscopía por medio del VNA.

Cada sensor está conformado por dos electrodos interdigitados en los cuales se deposita la muestra utilizando la plataforma microfluídica. Una vez que las cámaras correspondientes se llenan con el fluido a caracterizar se inicia el procedimiento de toma de datos con el VNA. En este caso, la técnica de caracterización por EIS se realiza a partir de los parámetros $S$ de red para cada sensor generados por el VNA. Sin embargo, al realizar las mediciones de parámetros $S$ en dispositivos integrados en un PCB (printed circuit board), la ruta física entre el puerto de conexión y el dispositivo bajo medición o DUT (device under test) puede generar efectos eléctricos no deseados que impiden la correcta caracterización por EIS requerida. Estos efectos son mayores conforme se aumenta la frecuencia de caracterización, por lo que a las frecuencias requeridas del orden de los $\mathrm{GHz}$ se convierte en una situación no favorable. Para solventar esta situación se utiliza una técnica de corrección conocida como de-embedding (extracción) [24]. Con esta técnica se miden los parámetros S de una topología en donde se 
elimina el DUT, siendo en este caso los electrodos. Por ello, junto a los sensores se fabricó un modelo de la estructura de acceso, pero sin los electrodos, tal y como se muestra en la figura 7. Los datos obtenidos por la prueba sin DUT se utilizan para aplicar un post procesamiento a las mediciones realizadas de las muestras acuosas y así se eliminan los efectos eléctricos no deseados que genera el sistema de conexión.

De esta forma, el procedimiento para generar el análisis de EIS consiste en medir los parámetros S a partir de las cámaras microfluídicas llenas del fluido en análisis, aplicar la técnica de extracción, convertir los nuevos parámetros $S$ a parámetros $Z$ y de estos se obtiene la impedancia característica presente en el sensor para el rango de frecuencias planteado.

\section{Llenado de cámaras y preparación de mediciones}

Para el procedimiento de llenado de las cámaras se utiliza un sistema bombeo que permite lograr un flujo de entrada constante regulable. Para la infusión del fluido muestra, se utiliza una velocidad o caudal no mayor a 0,02 $\mu \mathrm{l} / \mathrm{min}$; esto para evitar generar presiones elevadas en las distintas secciones que conforman el sistema de canales y cámaras del sistema microfluídico. Este proceso de llenado de cámaras se puede visualizar en la imagen de la figura 8.

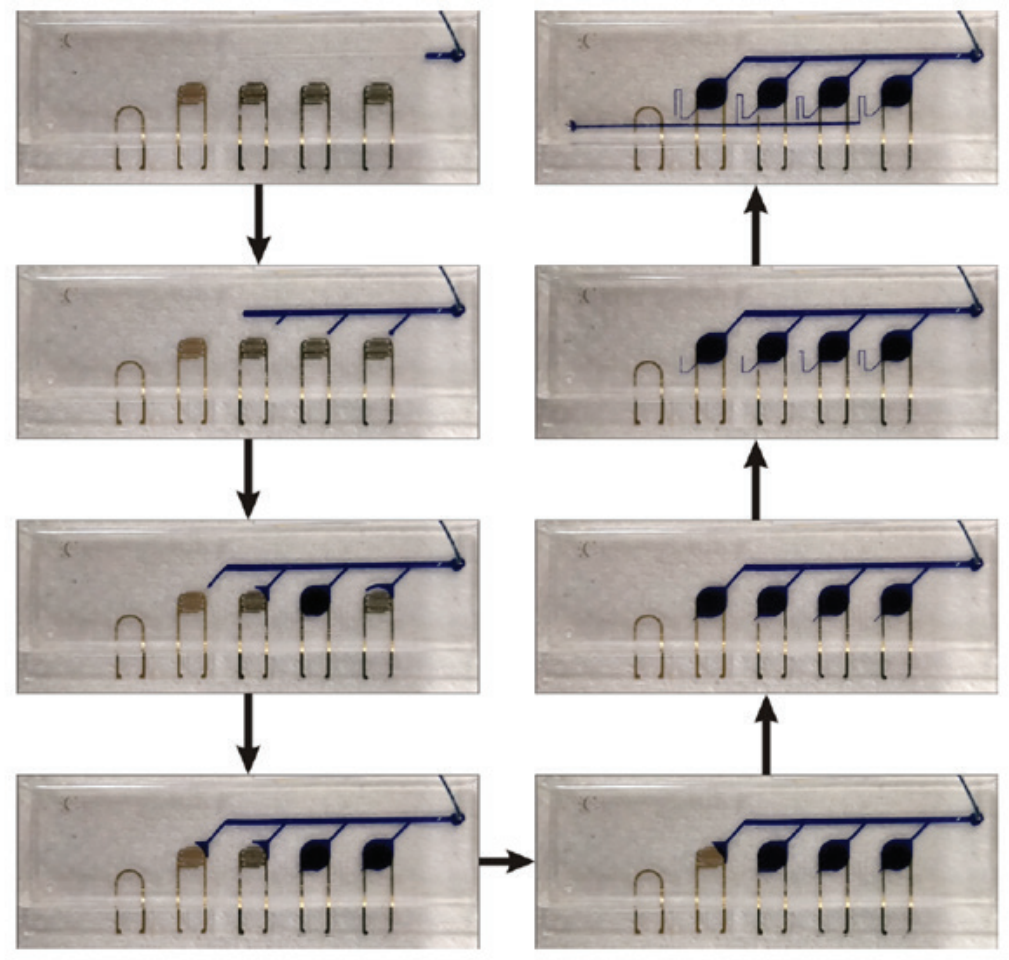

Figura 8. Ensayo de llenado de las cámaras con un fluido con color azul para su fácil visualización.

Con el procedimiento de caracterización de los electrodos y el de llenado de cámaras definidos, se tomaron diferentes muestras de fluido con el fin de aplicar la EIS y observar los resultados. Para ello se seleccionaron cuatro fluidos diferentes: airea, agua, acetona y alcohol isopropílico. En el cuadro 1 se muestra las constantes dieléctricas o permitividad de cada uno de estos fluidos seleccionados. 
Cuadro 1. Constante dieléctrica o permitividad relativa de las muestras utilizadas.

\begin{tabular}{|c|c|}
\hline Muestra & Permitividad relativa \\
\hline Aire & 1,00051 \\
\hline Alcohol Isopropílico & 18 \\
\hline Acetona & 21 \\
\hline Agua & 75,8 \\
\hline
\end{tabular}

El modelo físico del sensor utilizado es el de un capacitor eléctrico, donde el fluido se comporta como el material dieléctrico que une físicamente los dos electrodos del sensor. Debido a esto, es importante tener presente que en un capacitor de placas la capacitancia de este se define según la ecuación:

$$
C=\varepsilon_{0} \varepsilon_{r}(A / d)
$$

donde $\varepsilon_{0}$ es la permitividad del vacío, $\varepsilon_{r}$ la permitividad relativa del material dieléctrico, $A$ el área equivalente de las placas y $d$ la distancia de separación equivalente entre placas. Según lo anterior, la capacitancia es directamente proporcional a la permitividad relativa. Otro aspecto importante es que el capacitor presenta una menor magnitud de impedancia según aumente la frecuencia durante el barrido de EIS. Sin embargo, esta magnitud de impedancia está relacionada con la permitividad del dieléctrico de forma inversa, por lo que si la permitividad es mayor genera valores de magnitud de impedancia menores.

\section{Resultados y discusión}

Los resultados de EIS para las cuatro muestras mencionadas se muestran en la figura 9. La respuesta de magnitud y fase se muestran por separado y el barrido en frecuencia se realizó hasta $4 \mathrm{GHz}$. A partir de la impedancia característica de la figura 9 se obtuvo la capacitancia de cada muestra en el espectro analizado según la figura 10. Estos resultados muestran una relación correcta en relación con la permitividad relativa de las muestras de la tabla 1, en donde por ejemplo el agua que presenta la mayor permitividad tiene los valores de capacitancia mayores. Por otro lado, el aire que presenta la menor permitividad presenta la capacitancia menor. Estos resultados muestran una correcta correlación con las características físicas de las muestras y permite presentar un análisis de EIS hasta $4 \mathrm{GHz}$. Nótese que las resonancias que aparecen en la respuesta son efectos remanentes de las líneas de transmisión, las cuales son difíciles de remover en los puntos observados.

Además, en la figura 10 se muestran las capacitancias extraídas para las diferentes soluciones, utilizando dos prototipos del sistema distintos. Esto indica que las mediciones son reproducibles y se observa las mismas tendencias en la capacitancia extraída, a pesar de que la distribución de la capacitancia en función de la frecuencia puede variar debido a diferencias en el prototipo y las líneas de acceso. Se observa que el rango donde es más sencillo determinar diferencias entre las soluciones (por contraste capacitivo) está limitado a 2 GHz. 

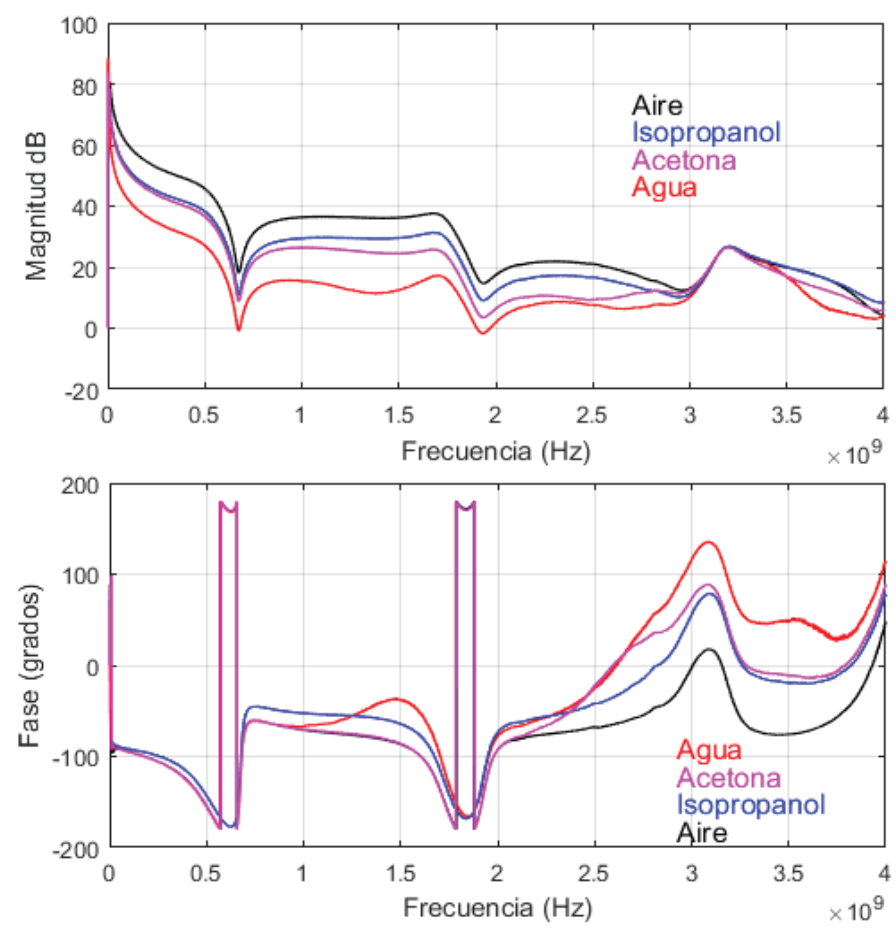

Figura 9. Impedancia característica obtenida del EIS para las cuatro muestras.
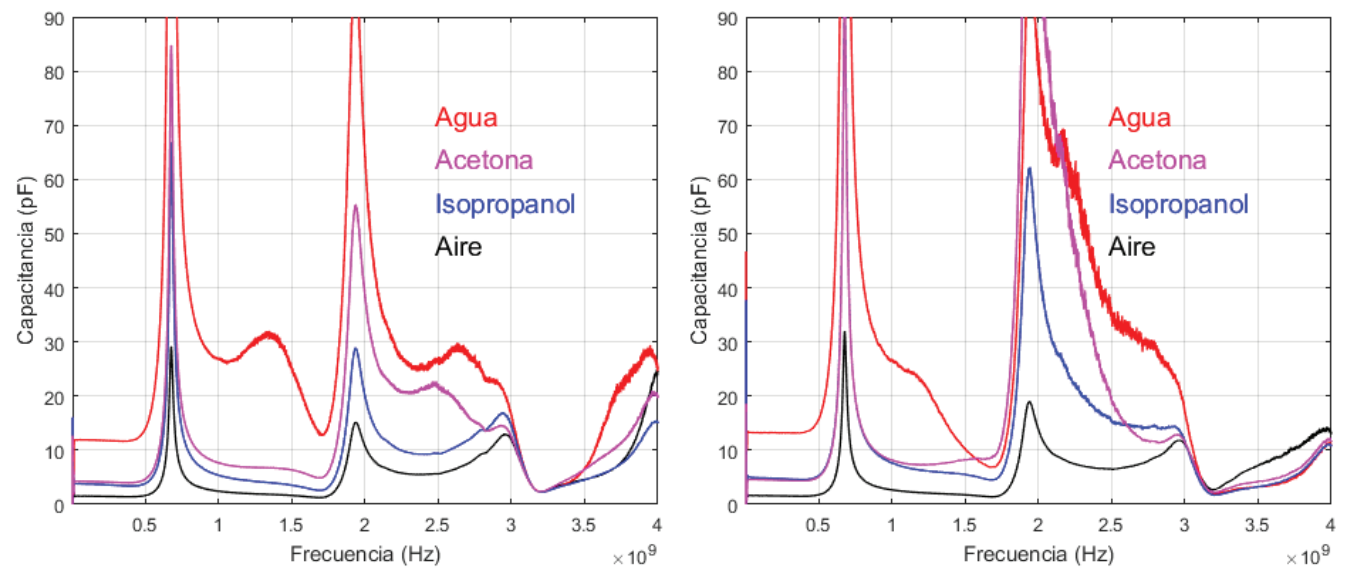

Figura 10. Capacitancia característica extraída para cada muestra analizada en dos sensores distintos.

\section{Conclusiones y recomendaciones}

En el presente artículo se ha descrito el proceso de diseño y desarrollo un prototipo de sistema para espectroscopía por impedancia eléctrica que consiste en un arreglo de cuatro sensores integrados en una plataforma microfluídica con una interfaz para mediciones eléctricas. Se destaca como logro de este trabajo la consolidación de una metodología para el diseño, simulación y fabricación de dispositivos de microfluídica básicos y que puede ser utilizada en combinación con interfaces electrónicas. Con el arreglo propuesto es posible distinguir entre diferentes soluciones por contraste capacitivo hasta una frecuencia de al menos $2 \mathrm{GHz}$, aunque por el tamaño de las cámaras es necesario remover el efecto de las líneas eléctricas de acceso utilizadas en las mediciones. Una de las principales características que potencian la capacidad de este sistema es el amplio espectro en frecuencia disponible, lo que permite 
brindar una herramienta base para investigaciones aplicadas que requieren estudios con EIS, como se menciona en [20] y [25]. Sin embargo, el prototipo desarrollado puede ser mejorado en diversos aspectos, entre ellos explorar el desarrollo de un sistema electrónico de menor costo en lugar del VNA para la adquisición de datos, la miniaturización del sensor para ampliar el rango de frecuencias, mejoras en el proceso de fabricación y más, trabajos que serán explorados en investigaciones futuras.

\section{Referencias}

[1] R. Meissner, "Impedance spectroscopy for in vitro toxicology", Ph.D. dissertation. Institut de Microtechnique, École Polytechnique Fédérale de Laussane, Switzerland, 2013.

[2] T. M. Curtis, J. Tabb, L. Romeo, S. J. Schwager, M. W. Widder, W. H. van der Schalie. "Improved cell sensitivity and longevity in a rapid impedance-based toxicity sensor", Journal of Applied Toxicology, 29(5):374-80, 2009.

[3] H. S. Kim, Y. H. Cho, A. B. Frazier, G. Chen, D. Shin, A. Han, "Whole cell impedance analysis of metastatic and non-metastatic cancer cells", in IEEE 22nd International Conference on Micro Electro Mechanical Systems, 2009, pp 399-402.

[4] Qiao, Y et al. (2012) Bioimpedance Analysis for the characterization of Breast Cancer Cells in Suspension (Vol. 59, No. 8, pp. 2332-2329). IEEE Transactions on biomedical engineering.

[5] Aberg, P. et al. Skin Cancer Identification Using Multifrequency Electrical Impedance_A Potential Screening Tool, IEEE Transactions on biomedical engineering, Vol. 51, No. 12, December 2004, pp. 2097-2102.

[6] O'Rourke, A. et al. Dielectric properties of human normal, malignant and cirrhotic liver tissue: in vivo and ex vivo measurements from 0.5 to $20 \mathrm{GHz}$ using a precision open-ended coaxial probe. Phys. Med. Biol. 52 (2007) 4707-4719

[7] Jäger, M. et al. Impedance Measurements in Cell Cultures on Polymer Slides. IEEE 2006 Electronics System Integration Technology Conference, pp. 1203-1208.

[8] Nacke, T. et al. Anwendungsmöglichkeiten der Impedanzspektroskopie in der Biotechnologie. Technisches Messen Plattform für Methoden, Systeme und Anwendungen der Messtechnik Vol. 69, Iss. 1/2002, pp. 12-18.

[9] Hautmann, S. y Müller, J. In-situ biomass characterization by impedance spectroscopy using a full-bridge circuit. Bioprocess and Biosystems Engineering 24, 2001, pp. 137-141.

[10] Asphahani, F. et al. Cellular Impedance Biosensors for Drug Screening and Toxin Detection. Analyst. 2007 September; 132(9): 835-841.

[11] Grenier et al. Recent Advances in Microwave-Based Dielectric Spectroscopy at the Cellular Level for Cancer Investigations. IEEE Transactions on Microwave theory and techniques, Vol. 61, No. 5, May 2013, pp 20232030.

[12] Grenier, K. et al. Microwave Dielectric Spectroscopy: an emerging analyzing technique for biological investigations at the cellular level. 2013 IEEE Topical Conference on Biomedical Wireless Technologies, Networks, and Sensing Systems (BioWireleSS), 2013, pp 40-42.

[13] S, Mobini, L. Leppik, and J.H. Barker, "Direct current electrical stimulation chamber for treating cells in vitro," Biotechniques, vol. 60, no. 2. 2018.

[14] R. García-Ramírez, et al., "Review on CMOS Circuit Architectures for Electrical Impedance Spectroscopy", in Proc. IEEE Central America and Panama Convention (Concapan 2016), San José, Costa Rica, november 9-11, 2016.

[15] R. García-Ramírez, et al. (2017), "A 0.13um CMOS Integrated Circuit for Electrical Impedance Spectroscopy from $1 \mathrm{kHz}$ to $10 \mathrm{GHz}$ " in Proc. IEEE 20th international System on Chip Conference, September 5-8, 2017, Hotel Novotel, Munich, Germany.

[16] Stanford University, "Designing your own device: Basic design rules," 2017.

[17] T. Miyazaki, J. Yang, S. Imamura, Y. Hirai, and K. Kamei, "Highly accurate measurement of trans-epithelial electrical resistance in organ-on-a-chip Institute for Integrated Cell-Material Sciences ( iCeMS ), Kyoto University , Kyoto , JAPAN and Faculty of Engineering , Kyoto University of Advanced Science, Kyoto," no. January, pp. 3-6, 2021.

[18] R. Munjal, F. Wendler, and O. Kanoun, "Embedded Wideband Measurement System for Fast Impedance Spectroscopy Using Undersampling," IEEE Trans. Instrum. Meas., vol. 69, no. 6, pp. 3461-3469, 2020. 
[19] R. Bounik, M. Gusmaroli, P. M. Misun, V. Viswam, A. Hierlemann, and M. M. Modena, "Integration of Discrete Sensors and Microelectrode Arrays into Open Microfluidic Hanging-Drop Networks," Proc. IEEE Int. Conf. Micro Electro Mech. Syst., vol. 2019-January, no. January, pp. 441-444, 2019.

[20] X. Ma, X. Du, H. Li, X. Cheng, and J. C. M. Hwang, "Ultra-wideband impedance spectroscopy of a live biological cell," IEEE Trans. Microw. Theory Tech., vol. 66, no. 8, pp. 3690-3696, 2018.

[21] Z. An, D. Kawashima, H. Obara, and M. Takei, "Extraction Method of Cell's Complex Permittivity in Cell Solutions from Measured Impedance by GHz Electrical Impedance Spectroscopy," IEEE Sens. J., vol. 21, no. 3, pp. 2505-2516, 2021.

[22] T. Chen, D. Dubuc, M. Poupot, J.-J. Fournie, and K. Grenier, Accurate nanoliter liquid characterization up to 40 ghz for biomedical applications: Toward noninvasive living cell monitoring," IEEE Transactions on Microwave Theory and Techniques, vol. 60, no. 12, pp. 4171-4177, 2012.

[23] S. Sugiura, J.-i. Edahiro, K. Kikuchi, K. Sumaru, and T. Kanamori, IPressure-driven perfusion culture microchamber array for a parallel drug cytotoxicity assay," Biotechnology and bioengineering, vol. 100, no. 6, pp. 11561165, 2008.

[24] Keysight Technologies, “@Keysight Technologies 2000-2020,” 177 2020. [Online]. Available: https://www.keysight.com/zz/en/assets/7018-06806/application-notes/5980-2784.pdf?success=true. [Accessed 159 2020].

[25] K. Grenier, U. De Toulouse, and F.- Toulouse, "Microfluidic On-Chip for Biomedical Applications," pp. 11-14, 2011. 